MSC 47J20, 49J40, 65K15, 90C25

\title{
ALGORITHM FOR VARIATIONAL INEQUALITY PROBLEM OVER THE SET OF SOLUTIONS THE EQUILIBRIUM PROBLEMS
}

\author{
Ya. I. Vedel, S. V. Denisov, V. V. Semenov
}

Faculty of Computer Science and Cybernetics, Taras Shevchenko Kiev National University, Kiev, Ukraine, E-mail: \{yana.vedel, sireukr, volodya.semenov\}@gmail.com

\section{АЛГОРИТМ ДЛЯ ВАРИАЦИОННОГО НЕРАВЕНСТВА НА МНОЖЕСТВЕ РЕШЕНИЙ ЗАДАЧИ О РАВНОВЕСИИ}

\author{
Я. И. ВЕДЕЛЬ, С. В. ДЕнисов, В. В. Семёнов
}

Факультет компьютерных наук и кибернетики, Киевский национальный университет имени Тараса Шевченко, Киев, Украина,

E-mail: \{yana.vedel, sireukr, volodya.semenov\}@gmail.com

\begin{abstract}
In this paper, we consider bilevel problem: variational inequality problem over the set of solutions the equilibrium problems. To solve this problem, an iterative algorithm is proposed that combines the ideas of a two-stage proximal method and iterative regularization. For monotone bifunctions of Lipschitz type and strongly monotone Lipschitz continuous operators, the theorem on strong convergence of sequences generated by the algorithm is proved.

KEYWORDS: bilevel problem, variational inequality, equilibrium problem, two-stage proximal algorithm, iterative regularization, strong convergence.
\end{abstract}

\begin{abstract}
АннотАция. Рассматривается двухуровневая задача: вариационное неравенство на множестве решений задачи о равновесии. Для решения данной задачи предложен итерационный алгоритм, сочетающий в себе идеи двухэтапного проксимального метода и итеративной регуляризации. Для монотонных бифункций липшицевого типа и сильно монотонных липшицевых операторов доказана теорема о сильной сходимости алгоритма.

КлЮчЕВЫЕ СловА: двухуровневая задача, вариационное неравенство, задача о равновесии, двухэтапный проксимальный метод, итеративная регуляризация, сильная сходимость.
\end{abstract}

\section{ВвеДЕНИЕ}

В оптимизации, теории некорректных задач распространен следующий прием решения задач с неединственным решением [1-3]. Задаче ставится в соответствие семейство возмущенных задач, однозначно и корректно разрешимых. Частное решение исходной задачи получают как предел решений 
возмущенных задач при уменьшении возмущений. Найденные таким образом частные решения удовлетворяют определенным дополнительным условиям. Например, минимальность нормы нормального решения оптимизационной задачи, полученного методом регуляризации А. Н. Тихонова.

С другой стороны, в исследовании операций возникают задачи оптимизации по последовательно заданным критериям (лексикографическая, последовательная или многоэтапная оптимизация). K настоящему времени известны приближенные методы одноэтапного решения этих задач, идейно близкие методам штрафа и регуляризации $[4,5]$.

В недавнее время возник интерес к последовательным (точнее, двухуровневым) вариационным неравенствам [6-8] и операторным включениям [9].

Одним из популярных направлений современного прикладного нелинейного анализа является исследование задач о равновесии (неравенств Ки Фаня, задач равновесного программирования) вида [10-17]

$$
\text { найти } x \in C: F(x, y) \geq 0 \quad \forall y \in C,
$$

где $C$ - непустое подмножество гильбертова пространства $H, F: C \times$ $C \rightarrow \mathbb{R}-$ функция со свойством $F(x, x)=0$ для всех $x \in C$ (называемая бифункцией). В виде (1) можно сформулировать задачи математического программирования, вариационные неравенства и многие игровые задачи.

В данной статье рассматривается двухуровневая задача: вариационное неравенство на множестве решений задачи о равновесии. Подобная задача рассматривалась в работе [15], где был предложен сильно сходящейся алгоритм, использовавший операцию вычисления значения резольвенты бифункции. Последнее существенно увеличивало трудоемкость алгоритма. Ниже для решения данной задачи будет предложен итерационный алгоритм, сочетающий в себе идеи двухэтапного проксимального метода $[16,17]$ и итеративной регуляризации [1]. Для монотонных бифункций липшицевого типа и сильно монотонных липшицевых операторов доказана теорема о сильной сходимости алгоритма.

\section{1. ПОСТАНОВКА ЗАДАЧИ}

Всюду далее $H$ - действительное гильбертово пространство со скалярным произведением $(\cdot, \cdot)$ и порожденной нормой $\|\cdot\|$.

Для оператора $A: H \rightarrow H$, множества $M \subseteq H$ и бифункции $F: H \times H \rightarrow$ $\mathbb{R}$ обозначим $V I(A, M)$ и $E P(F, M)$ множества

$$
\{x \in M:(A x, y-x) \geq 0 \quad \forall y \in M\} \quad \text { и }\{x \in M: F(x, y) \geq 0 \quad \forall y \in M\} \text {, }
$$
соответственно.

Для непустого выпуклого замкнутого множества $C \subseteq H$ рассмотрим двухуровневую задачу:

$$
\text { найти } x \in V I(A, E P(F, C)) \text {. }
$$

Будем предполагать выполненными следующие условия:

(A1) $F(x, x)=0$ для всех $x \in C$;

(A2) $F(x, y)+F(y, x) \leq 0$ для всех $x, y \in C$ (монотонность); 
(А3) для всех $x \in C$ функция $F(x, \cdot)$ полунепрерывна снизу и выпукла на $C$;

(А4) для всех $y \in C$ функция $F(\cdot, y)$ слабо полунепрерывна сверху на $C$;

(A5) для всех $x, y, z \in C$ имеет место

$$
F(x, y) \leq F(x, z)+F(z, y)+a\|x-z\|^{2}+b\|z-y\|^{2},
$$

где $a, b-$ положительные константы (липшицевость).

(A6) $E P(F, C) \neq \emptyset$.

(A7) $A: C \rightarrow H-\mu$-сильно монотонный и $L$-липшицевый оператор.

При данных условиях множество $E P(F, C)$ выпуклое и замкнутое [11], а задача (2) имеет единственное решение $x^{*} \in H[18]$.

Замечание 1. Условие (А5) типа липшицевости введено G. Mastroeni в [12]. Например, бифункция $F(x, y)=(A x, y-x)$ с липшицевым оператором $A: C \rightarrow H$ удовлетворяет (А5) с $a=\frac{L \varepsilon}{2}, b=\frac{L}{2 \varepsilon}$, где $\varepsilon>0$.

Напомним несколько фактов. Понадобится следующая известная лемма о рекуррентных числовых неравенствах [1].

Лемма 1. Пусть последовательность неотрицательных чисел $\xi_{n}$ удовлетворяет рекуррентному неравенству

$$
\xi_{n+1} \leq\left(1-\alpha_{n}\right) \xi_{n}+\beta_{n}
$$

где последовательности $\left(\alpha_{n}\right)$ и $\left(\beta_{n}\right)$ обладают свойствами:

1) $\alpha_{n} \in(0,1) m a \sum_{n=1}^{\infty} \alpha_{n}=+\infty$;

2) $\varlimsup_{n \rightarrow \infty} \frac{\beta_{n}}{\alpha_{n}} \leq 0$.

Тогда $\lim _{n \rightarrow \infty} \xi_{n}=0$.

Пусть $g: H \rightarrow \mathbb{R} \cup\{+\infty\}$ - собственная выпуклая полунепрерывная снизу функция. Проксимальным оператором [19], ассоциированным с функцией $g$, называют оператор

$$
H \ni x \mapsto \operatorname{prox}_{g} x=\operatorname{argmin}_{y \in \operatorname{dom} g}\left(g(y)+\frac{1}{2}\|y-x\|^{2}\right) \in \operatorname{dom} g .
$$

Оператор $\operatorname{prox}_{g}$ - твердо нерастягивающий (firmly nonexpansive) и

$$
g(y)-g(z)+(z-x, y-z) \geq 0 \forall y \in \operatorname{dom} g \Leftrightarrow z=\operatorname{prox}_{g} x .
$$

Замечание 2. Вышеприведенное наблюдение дает следующий критерий оптимальности [19]:

$$
x \in \operatorname{argmin} g \Leftrightarrow x=\operatorname{prox}_{g} x .
$$

\section{2. АППРОКСИМАЦИЯ ТИХОНОВА-БРАУДЕРА}

Рассмотрим вспомогательную задачу о равновесии:

$$
\text { найти } x \in C: \quad F(x, y)+\varepsilon(A x, y-x) \geq 0 \quad \forall y \in C,
$$

где $\varepsilon>0$. 
Следуя А. Б. Бакушинскому [1], назовем задачу (3) аппроксимацией Тихонова-Браудера двухуровневой задачи $(2)^{1}$. Из результатов [11] следует существование и единственность решения $x_{\varepsilon} \in C$ задачи (3) для любого $\varepsilon>0$.

Элементы $x_{\varepsilon} \in C$ имеют несколько важных свойств.

Лемма 2. Справедливы следующие неравенства:

(i) $\left\|x_{\varepsilon}\right\| \leq \frac{1}{\mu}\left\|A x^{*}\right\|+\left\|x^{*}\right\|$ для всех $\varepsilon>0$;

(ii) $\left\|x_{\varepsilon}-x_{\delta}\right\| \leq \frac{|\varepsilon-\delta|}{\varepsilon} \frac{1}{\mu}\left(1+\frac{L}{\mu}\right)\left\|A x^{*}\right\|$ для всех $\varepsilon, \delta>0$.

Доказательство. Пусть $\varepsilon>0$. Для $x_{\varepsilon}-$ решения задачи (3) и произвольного элемента $\hat{x} \in E P(F, C)$ имеем

$$
F\left(x_{\varepsilon}, \hat{x}\right)+\varepsilon\left(A x_{\varepsilon}, \hat{x}-x_{\varepsilon}\right) \geq 0 \text { и } F\left(\hat{x}, x_{\varepsilon}\right) \geq 0 .
$$

Сложив неравенства и воспользовавшись монотонностью бифункции $F$, получим

$$
\left(A x_{\varepsilon}, \hat{x}-x_{\varepsilon}\right) \geq 0
$$

то есть,

$$
\left(A x_{\varepsilon}-A \hat{x}, x_{\varepsilon}-\hat{x}\right) \leq\left(A \hat{x}, \hat{x}-x_{\varepsilon}\right) .
$$

Сильная монотонность оператора $A$ и неравенство Шварца дают

$$
\mu\left\|x_{\varepsilon}-\hat{x}\right\| \leq\|A \hat{x}\|,
$$

откуда и следует (i).

Докажем (ii). Пусть $x_{\varepsilon}$ и $x_{\delta}$ - решения задачи (3) с $\varepsilon>0$ и $\delta>0$, соответственно. Имеем

$$
F\left(x_{\varepsilon}, x_{\delta}\right)+\varepsilon\left(A x_{\varepsilon}, x_{\delta}-x_{\varepsilon}\right) \geq 0 \text { и } F\left(x_{\delta}, x_{\varepsilon}\right)+\delta\left(A x_{\delta}, x_{\varepsilon}-x_{\delta}\right) \geq 0 .
$$

Сложив неравенства и воспользовавшись монотонностью бифункции $F$, получим

$$
\varepsilon\left(A x_{\varepsilon}, x_{\delta}-x_{\varepsilon}\right)+\delta\left(A x_{\delta}, x_{\varepsilon}-x_{\delta}\right) \geq 0 .
$$

Перепишем последнее неравенство в виде

$$
\varepsilon\left(A x_{\varepsilon}-A x_{\delta}, x_{\varepsilon}-x_{\delta}\right) \leq(\delta-\varepsilon)\left(A x_{\delta}, x_{\varepsilon}-x_{\delta}\right) .
$$

Воспользовавшись сильной монотонностью оператора $A$, получим

$$
\varepsilon \mu\left\|x_{\varepsilon}-x_{\delta}\right\|^{2} \leq|\delta-\varepsilon|\left\|A x_{\delta}\right\|\left\|x_{\varepsilon}-x_{\delta}\right\|,
$$

то есть,

$$
\left\|x_{\varepsilon}-x_{\delta}\right\| \leq \frac{|\delta-\varepsilon|}{\varepsilon \mu}\left\|A x_{\delta}\right\|
$$

\footnotetext{
${ }^{1}$ Для решения экстремальных задач подобная аппроксимация была предложена А. Н. Тихоновым при построении регуляризующих алгоритмов, а позднее F. Browder $[2,3]$ применял похожую схему для устойчивой аппроксимации нормального решения вариационного неравенства или проекции заданной точки на множество неподвижных точек нерастягивающих операторов.
} 
Оценим сверху при помощи (4) норму $\left\|A x_{\delta}\right\|$

$$
\begin{aligned}
\left\|A x_{\delta}\right\| \leq\left\|A x^{*}\right\|+\left\|A x_{\delta}-A x^{*}\right\| & \leq \\
\leq & \left\|A x^{*}\right\|+L\left\|x_{\delta}-x^{*}\right\| \leq\left(1+\frac{L}{\mu}\right)\left\|A x^{*}\right\| .
\end{aligned}
$$

Использовав оценку (6) в (5) приходим к (ii).

При стремлении малого положительного параметра $\varepsilon$ к нулю элементы $x_{\varepsilon}$ сильно сходятся к решению задачи (2).

Лемма 3. Пусть выполняются условия (А1)-(A4) и (А6), (А7). Тогда

$$
\lim _{\varepsilon \rightarrow 0}\left\|x_{\varepsilon}-x^{*}\right\|=0 .
$$

Доказательство. В силу (i) леммы 2 из $\left\{x_{\varepsilon}\right\}_{\varepsilon>0}$ можно извлечь слабо сходящуюся к $w \in C$ последовательность $\left(x_{\varepsilon_{n}}\right)\left(\varepsilon_{n} \rightarrow 0\right)$. Воспользовавшись слабой полунепрерывностью сверху функции $F(\cdot, y)$, перейдем к пределу в

$$
F\left(x_{\varepsilon_{n}}, y\right)+\varepsilon_{n}\left(A x_{\varepsilon_{n}}, y-x_{\varepsilon_{n}}\right) \geq 0 \quad \forall y \in C .
$$

Получим

$$
F(w, y) \geq 0 \quad \forall y \in C,
$$

то есть, $w \in E P(F, C)$. А переходя в неравенстве

$$
\left(A \hat{x}, \hat{x}-x_{\varepsilon_{n}}\right) \geq\left(A x_{\varepsilon_{n}}, \hat{x}-x_{\varepsilon_{n}}\right) \geq 0 \quad \forall \hat{x} \in E P(F, C)
$$

к пределу, получим

$$
(A \hat{x}, \hat{x}-w) \geq 0 \quad \forall \hat{x} \in E P(F, C),
$$

то есть, $w=x^{*}$. Покажем, что

$$
\lim _{n \rightarrow \infty}\left\|x_{\varepsilon_{n}}-x^{*}\right\|=0
$$

Это следует из неравенства

$$
\mu\left\|x_{\varepsilon_{n}}-x^{*}\right\|^{2} \leq\left(A x_{\varepsilon_{n}}-A x^{*}, x_{\varepsilon_{n}}-x^{*}\right) \leq\left(A x^{*}, x^{*}-x_{\varepsilon_{n}}\right) .
$$

Из единствености элемента $x^{*}$ получаем $\lim _{\varepsilon \rightarrow 0}\left\|x_{\varepsilon}-x^{*}\right\|=0$.

Перейдем к описанию предлагаемого алгоритма решения двухуровневой задачи (2).

\section{3. Алгоритм}

В работе [16] для аппроксимации элементов множества $E P(F, C)$ был предложен алгоритм (названный двухэтапным проксимальным) вида

$$
\left\{\begin{array}{l}
y_{n}=\operatorname{prox}_{\lambda_{n} F\left(y_{n-1}, \cdot\right)} x_{n}, \\
x_{n+1}=\operatorname{prox}_{\lambda_{n} F\left(y_{n}, \cdot\right)} x_{n},
\end{array}\right.
$$

где $\lambda_{n}>0$. Отталкиваясь от схемы (7), для решения задачи (2) предлагаем следующий алгоритм. 
Алгоритм 1. Для $x_{1}, y_{0} \in C$ генерируем последовательность элементов $x_{n}, y_{n} \in C$ при помощи итерационной схемы

$$
\left\{\begin{array}{l}
z_{n}=x_{n}-\alpha_{n} \lambda_{n} A x_{n}, \\
y_{n}=\operatorname{prox}_{\lambda_{n} F\left(y_{n-1}, \cdot\right)} z_{n}=\operatorname{argmin}_{y \in C}\left(\lambda_{n} F\left(y_{n-1}, y\right)+\frac{1}{2}\left\|y-z_{n}\right\|^{2}\right), \\
x_{n+1}=\operatorname{prox}_{\lambda_{n} F\left(y_{n}, \cdot\right)} z_{n}=\operatorname{argmin}_{y \in C}\left(\lambda_{n} F\left(y_{n}, y\right)+\frac{1}{2}\left\|y-z_{n}\right\|^{2}\right),
\end{array}\right.
$$

где $\lambda_{n}>0, \alpha_{n}>0$.

На каждом шаге алгоритма 1 следует решить две выпуклые задачи с сильно выпуклыми функциями.

Относительно параметров алгоритма 1 будем предполагать выполненными следующие условия:

(B1) $\lambda_{n} \in[\underline{\lambda}, \bar{\lambda}] \subseteq\left(0, \frac{1}{2(2 a+b)}\right)$;

(B2) $\lim _{n \rightarrow \infty} \alpha_{n}=0$;

(B3) $\sum_{n=1}^{\infty} \alpha_{n}=+\infty$;

(B4) $\lim _{n \rightarrow \infty} \frac{\alpha_{n+1}-\alpha_{n}}{\alpha_{n}^{2}}=0$.

Замечание 3. В качестве подходящей последовательности $\left(\alpha_{n}\right)$ можно взять следующую:

$$
\alpha_{n}=\frac{1}{n^{p}}, \quad p \in(0,1)
$$

Замечание 4. Если $F(x, y)=(B x, y-x)$, то алгоритм 1 принимает вид:

$$
\left\{\begin{array}{l}
x_{1} \in C, y_{0} \in C \\
z_{n}=x_{n}-\alpha_{n} \lambda_{n} A x_{n} \\
y_{n}=P_{C}\left(z_{n}-\lambda_{n} B y_{n-1}\right) \\
x_{n+1}=P_{C}\left(z_{n}-\lambda_{n} B y_{n}\right)
\end{array}\right.
$$

где $P_{C}$ - оператор метрического проектирования на множество $C$. Данный метод при $A=I$ был изучен в [20]. А частный случай схемы

$$
\left\{\begin{array}{l}
y_{n}=P_{C}\left(z_{n}-\lambda_{n} B y_{n-1}\right) \\
x_{n+1}=P_{C}\left(z_{n}-\lambda_{n} B y_{n}\right)
\end{array}\right.
$$

был предложен в [21] для поиска седловых точек выпукло-вогнутых функций, определенных в конечномерном евклидовом пространстве. В работе [22] доказана сходимость этого алгоритма для вариационных неравенств с монотонными и липшицевыми операторами, действующими в бесконечномерном гильбертовом пространстве, а также предложена его экономичная модификация. В работах $[23,24]$ изучены варианты метода с использованием брэгмановского расстояния вместо евклидового.

Замечание 5. В [8] для двухуровневого вариационного неравенства был предложен и обоснован близкий алгоритм:

$$
\left\{\begin{array}{l}
x_{1} \in C \\
y_{n}=P_{C}\left(x_{n}-\lambda_{n} B x_{n}\right) \\
z_{n}=P_{C}\left(x_{n}-\lambda_{n} B y_{n}\right) \\
x_{n+1}=z_{n}-\alpha_{n} A z_{n}
\end{array}\right.
$$




\section{АЛГОРИТМ ДЛЯ ВАРИАЦИОННОГО НЕРАВЕНСТВА}

Алгоритм 1 сочетает в себе идеи двухэтапного проксимального метода $[16,17]^{2}$ и итеративной регуляризации [1]. Доказательство его сходимости проведем по следующей схеме. Пусть $x_{\alpha_{n}}$ - решение задачи (3) при $\varepsilon=\alpha_{n}$. Поскольку

$$
\left\|x_{n}-x^{*}\right\| \leq\left\|x_{n}-x_{\alpha_{n}}\right\|+\left\|x_{\alpha_{n}}-x^{*}\right\|, \quad \lim _{n \rightarrow \infty}\left\|x_{\alpha_{n}}-x^{*}\right\|=0,
$$

то достаточно показать, что порожденная алгоритмом 1 последовательность $\left(x_{n}\right)$ обладает свойством

$$
\lim _{n \rightarrow \infty}\left\|x_{n}-x_{\alpha_{n}}\right\|=0
$$

\section{4. ДОКАЗАТЕЛЬСТво СХОДИМОСТИ}

Доказательство сходимости алгоритма 1 начнем с доказательства важного неравенства для порождаемых им последовательностей $\left(x_{n}\right),\left(y_{n}\right)$ и элементов $x_{\alpha_{n}}$.

Лемма 4. Для порожденных алгоритмом 1 последовательностей $\left(x_{n}\right)$, $\left(y_{n}\right)$ и элементов $x_{\alpha_{n}}$ выполняется неравенство

$$
\begin{aligned}
& \left\|x_{n+1}-x_{\alpha_{n}}\right\|^{2} \leq\left(1-\alpha_{n} \lambda_{n} \mu\right)\left\|x_{n}-x_{\alpha_{n}}\right\|^{2}- \\
& -\left(1-4 \lambda_{n} a-\alpha_{n} \lambda_{n} \frac{L^{2}}{\mu}\right)\left\|x_{n}-y_{n}\right\|^{2}-\left(1-2 \lambda_{n} b\right)\left\|y_{n}-x_{n+1}\right\|^{2}+ \\
& +4 \lambda_{n} a\left\|y_{n-1}-x_{n}\right\|^{2} .
\end{aligned}
$$

Доказательство. Имеем

$$
\begin{gathered}
\left\|x_{n+1}-x_{\alpha_{n}}\right\|^{2}=\left\|x_{n}-x_{\alpha_{n}}\right\|^{2}-\left\|x_{n}-x_{n+1}\right\|^{2}+2\left(x_{n+1}-x_{n}, x_{n+1}-x_{\alpha_{n}}\right)= \\
=\left\|x_{n}-x_{\alpha_{n}}\right\|^{2}-\left\|x_{n}-y_{n}\right\|^{2}-\left\|y_{n}-x_{n+1}\right\|^{2}- \\
\quad-2\left(x_{n}-y_{n}, y_{n}-x_{n+1}\right)+2\left(x_{n+1}-x_{n}, x_{n+1}-x_{\alpha_{n}}\right) .
\end{gathered}
$$

Из определения точек $x_{n+1}$ и $y_{n}$ следует

$$
\begin{aligned}
& \lambda_{n} F\left(y_{n}, x_{\alpha_{n}}\right)-\lambda_{n} F\left(y_{n}, x_{n+1}\right) \geq\left(x_{n+1}-x_{n}+\alpha_{n} \lambda_{n} A x_{n}, x_{n+1}-x_{\alpha_{n}}\right), \\
& \lambda_{n} F\left(y_{n-1}, x_{n+1}\right)-\lambda_{n} F\left(y_{n-1}, y_{n}\right) \geq-\left(x_{n}-\alpha_{n} \lambda_{n} A x_{n}-y_{n}, y_{n}-x_{n+1}\right) .
\end{aligned}
$$

Использовав неравенства (10), (11) для оценки скалярных произведений в (9), получаем

$$
\begin{array}{r}
\left\|x_{n+1}-x_{\alpha_{n}}\right\|^{2} \leq\left\|x_{n}-x_{\alpha_{n}}\right\|^{2}-\left\|x_{n}-y_{n}\right\|^{2}-\left\|y_{n}-x_{n+1}\right\|^{2}+ \\
+2 \lambda_{n}\left\{F\left(y_{n}, x_{\alpha_{n}}\right)-F\left(y_{n}, x_{n+1}\right)+F\left(y_{n-1}, x_{n+1}\right)-F\left(y_{n-1}, y_{n}\right)\right\}+ \\
+2 \alpha_{n} \lambda_{n}\left(A x_{n}, x_{\alpha_{n}}-y_{n}\right) .
\end{array}
$$

\footnotetext{
2Заметим, что в последнее время вариант данного метода для вариационных неравенств стал известен в среде специалистов по машинному обучению (специализирующихся в обучении порождающих состязательных сетей) под названием «Extrapolation from the Past» [25].
} 
Липшицевость бифункции $F$ гарантирует выполнение неравенства

$$
\begin{aligned}
& -F\left(y_{n}, x_{n+1}\right)+F\left(y_{n-1}, x_{n+1}\right)-F\left(y_{n-1}, y_{n}\right) \leq \\
& \quad \leq a\left\|y_{n-1}-y_{n}\right\|^{2}+b\left\|y_{n}-x_{n+1}\right\|^{2} .
\end{aligned}
$$

Использовав вышеприведенную оценку в (12), получаем

$$
\begin{aligned}
\left\|x_{n+1}-x_{\alpha_{n}}\right\|^{2} & \leq\left\|x_{n}-x_{\alpha_{n}}\right\|^{2}-\left\|x_{n}-y_{n}\right\|^{2}-\left\|y_{n}-x_{n+1}\right\|^{2}+ \\
+ & 2 \lambda_{n} a\left\|y_{n-1}-y_{n}\right\|^{2}+2 \lambda_{n} b\left\|y_{n}-x_{n+1}\right\|^{2}+ \\
& +2 \alpha_{n} \lambda_{n}\left(A x_{n}, x_{\alpha_{n}}-y_{n}\right)+2 \lambda_{n} F\left(y_{n}, x_{\alpha_{n}}\right) .
\end{aligned}
$$

Член $\left\|y_{n-1}-y_{n}\right\|^{2}$ оценим следующим образом

$$
\left\|y_{n-1}-y_{n}\right\|^{2} \leq 2\left\|y_{n-1}-x_{n}\right\|^{2}+2\left\|y_{n}-x_{n}\right\|^{2} .
$$

Имеем

$$
\begin{aligned}
&\left\|x_{n+1}-x_{\alpha_{n}}\right\|^{2} \leq\left\|x_{n}-x_{\alpha_{n}}\right\|^{2}-\left\|x_{n}-y_{n}\right\|^{2}-\left\|y_{n}-x_{n+1}\right\|^{2}+ \\
&+4 \lambda_{n} a\left\|y_{n-1}-x_{n}\right\|^{2}+ 4 \lambda_{n} a\left\|y_{n}-x_{n}\right\|^{2}+2 \lambda_{n} b\left\|y_{n}-x_{n+1}\right\|^{2}+ \\
&+2 \alpha_{n} \lambda_{n}\left(A x_{n}, x_{\alpha_{n}}-y_{n}\right)+2 \lambda_{n} F\left(y_{n}, x_{\alpha_{n}}\right) .
\end{aligned}
$$

Из монотонности бифункции $F$ следует

$$
F\left(y_{n}, x_{\alpha_{n}}\right) \leq-F\left(x_{\alpha_{n}}, y_{n}\right),
$$

откуда

$$
F\left(y_{n}, x_{\alpha_{n}}\right)-\alpha_{n}\left(A x_{\alpha_{n}}, y_{n}-x_{\alpha_{n}}\right) \leq-F\left(x_{\alpha_{n}}, y_{n}\right)-\alpha_{n}\left(A x_{\alpha_{n}}, y_{n}-x_{\alpha_{n}}\right) .
$$

Поскольку

$$
F\left(x_{\alpha_{n}}, y_{n}\right)+\alpha_{n}\left(A x_{\alpha_{n}}, y_{n}-x_{\alpha_{n}}\right) \geq 0
$$

TO

$$
F\left(y_{n}, x_{\alpha_{n}}\right) \leq \alpha_{n}\left(A x_{\alpha_{n}}, y_{n}-x_{\alpha_{n}}\right) .
$$

Учтя последнюю оценку в (14), приходим к неравенству

$$
\begin{array}{r}
\left\|x_{n+1}-x_{\alpha_{n}}\right\|^{2} \leq\left\|x_{n}-x_{\alpha_{n}}\right\|^{2}-\left\|x_{n}-y_{n}\right\|^{2}-\left\|y_{n}-x_{n+1}\right\|^{2}+ \\
+4 \lambda_{n} a\left\|y_{n-1}-x_{n}\right\|^{2}+4 \lambda_{n} a\left\|y_{n}-x_{n}\right\|^{2}+2 \lambda_{n} b\left\|y_{n}-x_{n+1}\right\|^{2}+ \\
+2 \alpha_{n} \lambda_{n}\left(A x_{n}-A x_{\alpha_{n}}, x_{\alpha_{n}}-y_{n}\right) .
\end{array}
$$

Оценим сверху член $\left(A x_{n}-A x_{\alpha_{n}}, x_{\alpha_{n}}-y_{n}\right)$. Имеем

$$
\begin{gathered}
\left(A x_{n}-A x_{\alpha_{n}}, x_{\alpha_{n}}-y_{n}\right)=\left(A x_{n}-A x_{\alpha_{n}}, x_{\alpha_{n}}-x_{n}\right)+ \\
+\left(A x_{n}-A x_{\alpha_{n}}, x_{n}-y_{n}\right) \leq-\mu\left\|x_{\alpha_{n}}-x_{n}\right\|^{2}+L\left\|x_{n}-x_{\alpha_{n}}\right\|\left\|x_{n}-y_{n}\right\| \leq \\
\leq-\mu\left\|x_{\alpha_{n}}-x_{n}\right\|^{2}+\frac{\mu}{2}\left\|x_{n}-x_{\alpha_{n}}\right\|^{2}+\frac{L^{2}}{2 \mu}\left\|x_{n}-y_{n}\right\|^{2}= \\
=-\frac{\mu}{2}\left\|x_{n}-x_{\alpha_{n}}\right\|^{2}+\frac{L^{2}}{2 \mu}\left\|x_{n}-y_{n}\right\|^{2} .
\end{gathered}
$$


Из неравенств (15) и (16) получаем

$$
\begin{aligned}
& \left\|x_{n+1}-x_{\alpha_{n}}\right\|^{2} \leq\left(1-\alpha_{n} \lambda_{n} \mu\right)\left\|x_{n}-x_{\alpha_{n}}\right\|^{2}- \\
& \quad-\left(1-4 \lambda_{n} a-\alpha_{n} \lambda_{n} \frac{L^{2}}{\mu}\right)\left\|x_{n}-y_{n}\right\|^{2}-\left(1-2 \lambda_{n} b\right)\left\|y_{n}-x_{n+1}\right\|^{2}+ \\
& +4 \lambda_{n} a\left\|y_{n-1}-x_{n}\right\|^{2} .
\end{aligned}
$$

что и требовалось.

Докажем оценку, из которой будет следовать сходимость к нулю последовательностей $\left(\left\|x_{n}-x_{\alpha_{n}}\right\|\right)$ и $\left(\left\|y_{n-1}-x_{n}\right\|\right)$.

Лемма 5. Для порожденных алгоритмом 1 последовательностей $\left(x_{n}\right)$, $\left(y_{n}\right)$ и элементов $x_{\alpha_{n}}$ при больших $n$ выполняется неравенство

$$
\begin{aligned}
& \| x_{n+1}- x_{\alpha_{n+1}}\left\|^{2}+\frac{4 \lambda_{n+1} a}{1-\alpha_{n+1} \lambda_{n+1} \mu}\right\| y_{n}-x_{n+1} \|^{2} \leq \\
& \leq\left(1-\frac{\alpha_{n} \lambda_{n} \mu}{2}\right)\left(\left\|x_{n}-x_{\alpha_{n}}\right\|^{2}+\frac{4 \lambda_{n} a}{1-\alpha_{n} \lambda_{n} \mu}\left\|y_{n-1}-x_{n}\right\|^{2}\right)+ \\
&+\frac{2 M}{\lambda_{n} \mu} \frac{\left(\alpha_{n+1}-\alpha_{n}\right)^{2}}{\alpha_{n}^{3}}
\end{aligned}
$$

где $M=\frac{1}{\mu}\left(1+\frac{L}{\mu}\right)\left\|A x^{*}\right\|$.

Доказательство. Имеем

$$
\begin{gathered}
\left\|x_{n+1}-x_{\alpha_{n}}\right\|^{2}=\left\|x_{n+1}-x_{\alpha_{n+1}}\right\|^{2}+\left\|x_{\alpha_{n+1}}-x_{\alpha_{n}}\right\|^{2}+ \\
+2\left(x_{n+1}-x_{\alpha_{n+1}}, x_{\alpha_{n+1}}-x_{\alpha_{n}}\right) \geq\left\|x_{n+1}-x_{\alpha_{n+1}}\right\|^{2}+ \\
+\left\|x_{\alpha_{n+1}}-x_{\alpha_{n}}\right\|^{2}-2\left\|x_{n+1}-x_{\alpha_{n+1}}\right\|\left\|x_{\alpha_{n+1}}-x_{\alpha_{n}}\right\| \geq \\
\quad \geq(1-\varepsilon)\left\|x_{n+1}-x_{\alpha_{n+1}}\right\|^{2}+\frac{(\varepsilon-1)}{\varepsilon}\left\|x_{\alpha_{n+1}}-x_{\alpha_{n}}\right\|^{2},
\end{gathered}
$$

где $\varepsilon>0$. Положим в (18) $\varepsilon=\frac{1}{2} \alpha_{n} \lambda_{n} \mu$. Получим

$$
\begin{aligned}
\left\|x_{n+1}-x_{\alpha_{n}}\right\|^{2} \geq \frac{2-\alpha_{n} \lambda_{n} \mu}{2} \| x_{n+1}- & x_{\alpha_{n+1}} \|^{2}- \\
& -\frac{2-\alpha_{n} \lambda_{n} \mu}{\alpha_{n} \lambda_{n} \mu}\left\|x_{\alpha_{n+1}}-x_{\alpha_{n}}\right\|^{2} .
\end{aligned}
$$

В силу правил согласования значений параметров $\alpha_{n}, \lambda_{n}$ при больших $n$ имеем $1-\alpha_{n} \lambda_{n} \mu>0$. С учетом второго неравенства леммы 2 из (19) выводим

$$
\begin{aligned}
\left\|x_{n+1}-x_{\alpha_{n}}\right\|^{2} \geq \frac{2-\alpha_{n} \lambda_{n} \mu}{2}\left\|x_{n+1}-x_{\alpha_{n+1}}\right\|^{2}- & \\
& \quad-\frac{\left(2-\alpha_{n} \lambda_{n} \mu\right)}{\alpha_{n} \lambda_{n} \mu} \frac{\left(\alpha_{n+1}-\alpha_{n}\right)^{2}}{\alpha_{n}^{2}} \frac{1}{\mu}\left(1+\frac{L}{\mu}\right)\left\|A x^{*}\right\|,
\end{aligned}
$$


для всех $n \geq n_{0}$. Использовав (20) в (8), получим (для $n \geq n_{0}$ )

$$
\begin{aligned}
& \frac{2-\alpha_{n} \lambda_{n} \mu}{2}\left\|x_{n+1}-x_{\alpha_{n+1}}\right\|^{2} \leq\left(1-\alpha_{n} \lambda_{n} \mu\right)\left\|x_{n}-x_{\alpha_{n}}\right\|^{2}- \\
& -\left(1-4 \lambda_{n} a-\alpha_{n} \lambda_{n} \frac{L^{2}}{\mu}\right)\left\|x_{n}-y_{n}\right\|^{2}-\left(1-2 \lambda_{n} b\right)\left\|y_{n}-x_{n+1}\right\|^{2}+ \\
& \quad+4 \lambda_{n} a\left\|y_{n-1}-x_{n}\right\|^{2}+\frac{\left(2-\alpha_{n} \lambda_{n} \mu\right)}{\alpha_{n} \lambda_{n} \mu} \frac{\left(\alpha_{n+1}-\alpha_{n}\right)^{2}}{\alpha_{n}^{2}} M
\end{aligned}
$$

где $M=\frac{1}{\mu}\left(1+\frac{L}{\mu}\right)\left\|A x^{*}\right\|$. Откуда следует неравенство

$$
\begin{aligned}
& \left\|x_{n+1}-x_{\alpha_{n+1}}\right\|^{2} \leq \frac{2-2 \alpha_{n} \lambda_{n} \mu}{2-\alpha_{n} \lambda_{n} \mu}\left\|x_{n}-x_{\alpha_{n}}\right\|^{2}- \\
& -\frac{2\left(1-4 \lambda_{n} a-\alpha_{n} \lambda_{n} \frac{L^{2}}{\mu}\right)}{2-\alpha_{n} \lambda_{n} \mu}\left\|x_{n}-y_{n}\right\|^{2}-\frac{2\left(1-2 \lambda_{n} b\right)}{2-\alpha_{n} \lambda_{n} \mu}\left\|y_{n}-x_{n+1}\right\|^{2}+ \\
& \quad+\frac{8 \lambda_{n} a}{2-\alpha_{n} \lambda_{n} \mu}\left\|y_{n-1}-x_{n}\right\|^{2}+\frac{2 M}{\lambda_{n} \mu} \frac{\left(\alpha_{n+1}-\alpha_{n}\right)^{2}}{\alpha_{n}^{3}}
\end{aligned}
$$

Перегруппировав члены в (21), получим

$$
\begin{gathered}
\left\|x_{n+1}-x_{\alpha_{n+1}}\right\|^{2}+\frac{4 \lambda_{n+1} a}{1-\alpha_{n+1} \lambda_{n+1} \mu}\left\|y_{n}-x_{n+1}\right\|^{2} \leq \\
\leq \frac{2-2 \alpha_{n} \lambda_{n} \mu}{2-\alpha_{n} \lambda_{n} \mu}\left(\left\|x_{n}-x_{\alpha_{n}}\right\|^{2}+\frac{4 \lambda_{n} a}{1-\alpha_{n} \lambda_{n} \mu}\left\|y_{n-1}-x_{n}\right\|^{2}\right)- \\
\quad-\frac{2\left(1-4 \lambda_{n} a-\alpha_{n} \lambda_{n} \frac{L^{2}}{\mu}\right)}{2-\alpha_{n} \lambda_{n} \mu}\left\|x_{n}-y_{n}\right\|^{2}- \\
-\left(\frac{1-2 \lambda_{n} b}{1-\frac{\alpha_{n} \lambda_{n} \mu}{2}}-\frac{4 \lambda_{n+1} a}{1-\alpha_{n+1} \lambda_{n+1} \mu}\right)\left\|y_{n}-x_{n+1}\right\|^{2}+ \\
+\frac{2 M}{\lambda_{n} \mu} \frac{\left(\alpha_{n+1}-\alpha_{n}\right)^{2}}{\alpha_{n}^{3}} .
\end{gathered}
$$

Поскольку $\lambda_{n} \in[\underline{\lambda}, \bar{\lambda}] \subseteq\left(0, \frac{1}{2(2 a+b)}\right)$ и $\alpha_{n}>0, \lim _{n \rightarrow \infty} \alpha_{n}=0$, то начиная с некоторого номера $n_{1}$ будут выполняться неравенства

$$
\frac{1-4 \lambda_{n} a-\alpha_{n} \lambda_{n} \frac{L^{2}}{\mu}}{2-\alpha_{n} \lambda_{n} \mu}>0, \quad \frac{1-2 \lambda_{n} b}{1-\frac{\alpha_{n} \lambda_{n} \mu}{2}}-\frac{4 \lambda_{n+1} a}{1-\alpha_{n+1} \lambda_{n+1} \mu}>0,
$$

и

$$
\frac{2-2 \alpha_{n} \lambda_{n} \mu}{2-\alpha_{n} \lambda_{n} \mu}<1-\frac{\alpha_{n} \lambda_{n} \mu}{2}
$$


Таким образом, для $n \geq N=\max \left\{n_{0}, n_{1}\right\}$ из (22) следует

$$
\begin{aligned}
\left\|x_{n+1}-x_{\alpha_{n+1}}\right\|^{2}+\frac{4 \lambda_{n+1} a}{1-\alpha_{n+1} \lambda_{n+1} \mu}\left\|y_{n}-x_{n+1}\right\|^{2} \leq & \\
\leq\left(1-\frac{\alpha_{n} \lambda_{n} \mu}{2}\right)\left(\left\|x_{n}-x_{\alpha_{n}}\right\|^{2}+\frac{4 \lambda_{n} a}{1-\alpha_{n} \lambda_{n} \mu}\left\|y_{n-1}-x_{n}\right\|^{2}\right)+ & +\frac{2 M}{\lambda_{n} \mu} \frac{\left(\alpha_{n+1}-\alpha_{n}\right)^{2}}{\alpha_{n}^{3}},
\end{aligned}
$$

что и требовалось доказать.

Сформулируем основной результат.

Теорема 1. Пусть выполняются условия (A1)-(A7) и (B1)-(B4). Тогда для порожденных алгоритмом 1 последовательностей $\left(x_{n}\right),\left(y_{n}\right)$ имеет место

$$
\lim _{n \rightarrow \infty}\left\|x_{n}-x^{*}\right\|=\lim _{n \rightarrow \infty}\left\|y_{n}-x^{*}\right\|=0,
$$

где $x^{*} \in H-$ единственное решение задачи (2).

Доказательство. В силу леммы 1 и неравенства (17) имеем

$$
\lim _{n \rightarrow \infty}\left(\left\|x_{n}-x_{\alpha_{n}}\right\|^{2}+\frac{4 \lambda_{n} a}{1-\alpha_{n} \lambda_{n} \mu}\left\|y_{n-1}-x_{n}\right\|^{2}\right)=0 .
$$

Откуда

$$
\lim _{n \rightarrow \infty}\left\|x_{n}-x_{\alpha_{n}}\right\|=\lim _{n \rightarrow \infty}\left\|y_{n-1}-x_{n}\right\|=0 .
$$

Из неравенства

$$
\left\|x_{n}-x^{*}\right\| \leq\left\|x_{n}-x_{\alpha_{n}}\right\|+\left\|x_{\alpha_{n}}-x^{*}\right\|,
$$

леммы 3 и (24) получаем искомые равенства (23).

Замечание 6. При $A=I$ задача $(2)$ совпадает с задачей поиска $P_{E P(F, C)} 0$. Таким образом, в этом случае алгоритм 1 является сильно сходящейся схемой вычисления нормального решения задачи о равновесии.

\section{ЗАКЛЮЧЕНИЕ}

В статье рассматривалась двухуровневая задача: вариационное неравенство на множестве решений задачи о равновесии. Для решения данной задачи предложен итерационный алгоритм, сочетающий в себе идеи двухэтапного проксимального метода $[16,17]$ и итеративной регуляризации [1]. Для монотонных бифункций липшицевого типа и сильно монотонных липшицевых операторов доказана теорема о сильной сходимости алгоритма.

В одной из ближайших работ мы планируем рассмотреть более специальный вариант алгоритма с адаптивным подбором параметра в проксимальном операторе.

Работа выполнена при поддержке Министерства образования и науки Украины (проект «Математичне моделювання та оптимізація динамічних систем для оборони, медицини та екології», 0219U008403) и Национальной 
академии наук Украины (проект «Нові методи дослідження коректності та розв'язання задач дискретної оптимізації, варіаційних нерівностей та їх застосування», 0119U101608).

\section{ЛитеРАТУРА}

1. Bakushinskii A. B., Goncharskii A. V. Iterative Methods for Solving Ill-Posed Problems. Moscow: Nauka, 1989. 126 p.

2. Browder F. Existence and approximation of solutions of nonlinear variational inequalities. Proc. Nat. Acad. Sci. USA. 1966. Vol. 56. No. 4. P. 1080-1086.

3. Browder F. E. Convergence of approximants of fixed points of nonexpansive nonlinear mappings in Banach spaces. Arch. Rational Mech. Anal. 1967. Vol. 24. P. 82-90.

4. Solodov M. An explicit descent method for bilevel convex optimization. Journal of Convex Analysis. 2007. Vol. 14. P. 227-238.

5. Voitova T. A., Denisov S. V., Semenov V. V. Alternating proximal algorithm for the problem of bilevel convex minimization. Reports of the National Academy of Sciences of Ukraine. 2012. No. 2. P. 56-62. (In Ukrainian)

6. Semenov V. V. About convergence of methods for solving bilevel variational inequalities with monotone operators. J. Num. Appl. Math. 2010. No. 2 (101). P. 121-129. (In Russian)

7. Denisov S. V., Semenov V. V. Proximal algorithm for bilevel variational inequalities: strong convergence. J. Num. Appl. Math. 2011. No. 3 (106). P. 27-32. (In Ukrainian)

8. Apostol R. Ya., Grynenko A. A., Semenov V.V. Iterative algorithms for monotone bilevel variational inequalities. J. Num. Appl. Math. 2012. No. 1 (107). P. 3-14. (In Ukrainian)

9. Voitova T. A., Semenov V. V. Method for solving the bilevel operator inclusions. J. Num. Appl. Math. 2010. No. 3 (102). P. 34-39. (In Russian)

10. Antipin A. S. Equilibrium programming: Proximal methods. Comput. Math. Math. Phys. 1997. Vol. 37. P. 1285-1296.

11. Combettes P. L., Hirstoaga S. A. Equilibrium Programming in Hilbert Spaces. J. Nonlinear Convex Anal. 2005. Vol. 6. P. 117-136.

12. Mastroeni G. On auxiliary principle for equilibrium problems. In: Daniele, P. et al. (eds.) Equilibrium Problems and Variational Models. Kluwer Academic Publishers, Dordrecht, 2003. P. 289-298.

13. Quoc T. D., Muu L. D., Hien N. V. Extragradient algorithms extended to equilibrium problems. Optimization. 2008. Vol. 57. P. 749-776.

14. Lyashko S. I., Semenov V. V., Voitova T. A. Low-cost modification of Korpelevich's methods for monotone equilibrium problems. Cybernetics and Systems Analysis. 2011. Vol. 47. P. 631-639.

15. Semenov V. V. Strongly Convergent Algorithms for Variational Inequality Problem Over the Set of Solutions the Equilibrium Problems. In: Zgurovsky M.Z. and Sadovnichiy V.A. (eds.) Continuous and Distributed Systems. Solid Mechanics and Its Applications, vol. 211, Springer International Publishing Switzerland, 2014. P. 131-146.

16. Lyashko S. I., Semenov V. V. A New Two-Step Proximal Algorithm of Solving the Problem of Equilibrium Programming. In: B. Goldengorin (ed.) Optimization 
and Its Applications in Control and Data Sciences. Springer Optimization and Its Applications, vol. 115. Springer, Cham, 2016. P. 315-325.

17. Chabak L., Semenov V., Vedel Y. A New Non-Euclidean Proximal Method for Equilibrium Problems. In: Chertov O., Mylovanov T., Kondratenko Y., Kacprzyk J., Kreinovich V., Stefanuk V. (eds.) Recent Developments in Data Science and Intelligent Analysis of Information. ICDSIAI 2018. Advances in Intelligent Systems and Computing, vol. 836. Springer, Cham, 2019. P. 50-58.

18. Kinderlehrer D. Stampacchia G. An introduction to variational inequalities and their applications. New York: Academic Press, 1980. Russian transl., Moscow: Mir, 1983. $256 \mathrm{p}$.

19. Bauschke H. H., Combettes P. L. Convex Analysis and Monotone Operator Theory in Hilbert Spaces. Berlin, Heidelberg, New York: Springer, 2011. 408 p.

20. Popov L. D. On schemes for the formation of a master sequence in a regularized extragradient method for solving variational inequalities. Russian Mathematics. 2004. Vol. 48. Issue 1. P. 67-76.

21. Popov L. D. A modification of the Arrow-Hurwicz method for search of saddle points. Mathematical notes of the Academy of Sciences of the USSR. 1980. Vol. 28. Issue 5. P. 845-848.

22. Malitsky Yu. V., Semenov V. V. An extragradient algorithm for monotone variational inequalities. Cybernetics and Systems Analysis. 2014. Vol. 50. P. 271-277.

23. Semenov V. V. A Version of the Mirror descent Method to Solve Variational Inequalities. Cybernetics and Systems Analysis. 2017. Vol. 53. P. 234-243.

24. Nomirovskii D. A., Rublyov V. V., Semenov V. V. Convergence of Two-Stage Method with Bregman Divergence for Solving Variational Inequalities. Cybernetics and Systems Analysis. 2019. Vol. 55. P. 359-368.

25. Gidel G., Berard H., Vincent P., Lacoste-Julien S. A Variational Inequality Perspective on Generative Adversarial Networks. arXiv:1802.10551. 2018.

Поступила: 01.04.2020 / Принята: 09.05.2020

\title{
АЛГОРИТМ ДЛЯ ВАРІАЦІЙНОЇ НЕРІВНОСТІ НА МНОЖИНІ РОЗВ'ЯЗКІВ ЗАДАЧІ ПРО РІВНОВАГУ
}

\author{
Я. І. Ведель, С. В. ДЕнисов, В. В. Семенов
}

Факультет комп'ютерних наук і кібернетики, Київський національний університет імені Тараса Шевченка, Київ, Україна, E-mail: \{yana.vedel, sireukr, volodya.semenov\}@gmail.com

АнотАція. Розглядається дворівнева задача: варіаційна нерівність на множині розв'язків задачі про рівновагу. Для розв'язання даної задачі запропоновано ітераційний алгоритм, що суміщає у собі ідеї двоетапного проксимального методу та ітеративної регуляризації. Для монотонних біфункцій ліпшицевого типу та сильно монотонних ліпшицевих операторів доведено теорему про сильну збіжність алгоритму.

Ключові словА: дворівнева задача, варіаційна нерівність, задача про рівновагу, двоетапний проксимальний метод, ітеративна регуляризація, сильна збіжність. 